PROCEEDINGS OF THE

AMERICAN MATHEMATICAL SOCIETY

Volume 126, Number 9, September 1998, Pages 2615-2617

S $0002-9939(98) 04339-1$

\title{
LINEAR CONSTITUENTS OF CERTAIN CHARACTER RESTRICTIONS
}

\author{
I. M. ISAACS AND G. R. ROBINSON \\ (Communicated by Ronald M. Solomon)
}

\begin{abstract}
Let $G$ be a finite irreducible complex linear group with $p$-power degree, where $p$ is a prime number. Then every $p^{\prime}$-subgroup of $G$ that is normalized by a Sylow $p$-subgroup must be abelian. This and related results are proved using an elementary character-theoretic argument.
\end{abstract}

Let $\chi \in \operatorname{Irr}(G)$, where $G$ is a finite group, and suppose $N$ is a subgroup whose order is relatively prime to the degree $\chi(1)$. If $N \triangleleft G$, then the irreducible constituents of the restriction $\chi_{N}$ are linear, since the degree of any such constituent divides both $\chi(1)$ and $|N|$. The main result of this note is that the condition that $N \triangleleft G$ can be considerably weakened without affecting the conclusion that the irreducible constituents of $\chi_{N}$ are linear. Instead of requiring that $N \triangleleft G$, it suffices to assume that the index $\left|G: \mathbf{N}_{G}(N)\right|$ is relatively prime to $\chi(1)$.

Theorem A. Let $\chi \in \operatorname{Irr}(G)$ and suppose $N \triangleleft L \subseteq G$. If $\operatorname{gcd}(\chi(1),|N||G: L|)=1$, then the irreducible constituents of $\chi_{N}$ are linear, and they are all conjugate under the action of $L$.

An immediate consequence is the following striking (but known) fact about linear groups.

Corollary B. Let $G$ be a finite irreducible complex linear group of p-power degree, where $p$ is a prime number. Let $P \in \operatorname{Syl}_{p}(G)$, and suppose that $P$ normalizes some $p^{\prime}$-subgroup $N \subseteq G$. Then $N$ is abelian.

Proof. Let $\chi$ be the given faithful irreducible character of $G$ having $p$-power degree. Let $L=N P$ and note that $\operatorname{gcd}(\chi(1),|N||G: L|)=1$. By Theorem A, the irreducible constituents of $\chi_{N}$ are linear, and it follows that $N$ is abelian.

Corollary B appeared as Corollary 9 of [1], where it was proved using indecomposable modules over $p$-local rings. It seems that the argument given here, deriving the result from Theorem A, is more direct and straightforward. This method is also more general, of course, and it yields the following, which is also immediate from Theorem A.

Corollary C. Let $G$ be a finite irreducible complex linear group of degree $n$, and write $\pi$ to denote the set of prime divisors of $n$. If $L \subseteq G$ has $\pi^{\prime}$-index, then $\mathbf{O}_{\pi^{\prime}}(L)$ is abelian.

Received by the editors February 14, 1997.

1991 Mathematics Subject Classification. Primary 20C15.

(C)1998 American Mathematical Society 
Theorem A is a consequence of the following general result.

Theorem D. Let $\chi \in \operatorname{Irr}(G)$ and suppose $N \triangleleft L \subseteq G$. Choose an irreducible constituent $\mu$ of $\chi_{N}$ and write $\chi_{L}=\psi+\eta$, where each irreducible constituent of $\psi$ lies over $\mu$ and no constituent of $\eta$ lies over $\mu$. Then the rational number

$$
\alpha=\frac{|N||G: L| \psi(1)}{\chi(1) \mu(1)}
$$

is actually an integer.

Proof. Let $e$ be the central idempotent corresponding to $\mu$ in the complex group algebra $\mathbb{C} N$. Since the coefficient of $n \in N$ in $e$ is $(\mu(1) /|N|) \mu\left(n^{-1}\right)$, we see that $|N| e / \mu(1)$ is an algebraic integer linear combination of the elements of $N$.

Let $f$ be the sum of the distinct $L$-conjugates of $e$, and note that $f$ is central in $\mathbb{C} L$. Also, if $\nu \in \operatorname{Irr}(N)$, then $\nu(f)$ is nonzero if and only if $\nu$ is $L$-conjugate to $\mu$, and in that case, $\nu(f)=\nu(1)$. Since all irreducible constituents of $\psi_{N}$ are conjugate to $\mu$ while none of the irreducible constituents of $\eta_{N}$ are conjugate to $\mu$, we see that

$$
\chi(f)=\psi(f)=\psi(1) .
$$

Now write $u=\sum t^{-1} f t$, where $t$ runs over a transversal for the right cosets of $L$ in $G$, and note that $u$ is centralized by $G$ since $f$ is centralized by $L$. Also, because $u$ is a sum of conjugates of $e$, it follows that $|N| u / \mu(1)$ is an algebraic integer combination of elements of $G$, and thus this central element of $\mathbb{C} G$ is an algebraic integer combination of class sums of $G$.

For each class sum $s$ in $\mathbb{C} G$, we know that $\chi(s) / \chi(1)=\omega_{\chi}(s)$ is an algebraic integer, and it follows that

$$
\frac{\chi(|N| u / \mu(1))}{\chi(1)}=\frac{|N| \chi(u)}{\chi(1) \mu(1)}=\frac{|N||G: L| \chi(f)}{\chi(1) \mu(1)}=\frac{|N||G: L| \psi(1)}{\chi(1) \mu(1)}=\alpha
$$

is an algebraic integer. This completes the proof.

We can now prove a slightly stronger version of Theorem A, where we relax the condition that $\chi(1)$ and $|N||G: L|$ are relatively prime. Theorem A is immediate from the following by setting $d=1$.

Theorem E. Let $\chi \in \operatorname{Irr}(G)$ and suppose that $N \triangleleft L \subseteq G$. Then every irreducible constituent $\mu$ of $\chi_{N}$ satisfies $\mu(1) \leq d$, where $d=\operatorname{gcd}(\chi(1),|N||G: L|)$. Furthermore, all irreducible constituents $\mu$ of $\chi_{N}$ for which $\mu(1)>d / 2$ are conjugate under the action of $L$.

Proof. As in Theorem D, write $\chi_{L}=\psi+\eta$, where all irreducible constituents of $\psi$ lie over $\mu$ and no irreducible consituent of $\eta$ lies over $\mu$. Now $\psi(1) / \mu(1)$ is an integer, and by Theorem $\mathrm{D}$, we know that $\chi(1)$ divides $|N||G: L|(\psi(1) / \mu(1))$. It follows that $\chi(1) / d$ divides $\psi(1) / \mu(1)$, and thus $\psi(1) \geq(\mu(1) / d) \chi(1)$. We know that $\psi(1) \leq \chi(1)$, however, and it follows that $\mu(1) \leq d$.

If $\mu(1)>d / 2$, then $\psi(1)>\chi(1) / 2$, and the sum of all $L$-conjugates of $\mu$ accounts for more than half of the degree of $\chi$. There can thus be at most one $L$-orbit of irreducible constituents of $\chi_{N}$ have degrees exceeding $d / 2$. 


\section{REFERENCES}

[1] I. M. Isaacs and L. Scott, Blocks and subgroups, J. of Algebra, 20 (1972) 630-636. MR 45:6944

Department of Mathematics, University of Wisconsin, Madison, Wisconsin 53706

E-mail address: isaacs@math.wisc.edu

Department of Mathematics, University of Leicester, Leicester LE1 7RH, England

E-mail address: grr1@mcs.le.ac.uk 